\title{
The Low Energy Dynamics of Non-BPS Branes
}

\author{
N.D. Lambert ${ }^{1}$ and I. Sachs ${ }^{2}$ \\ ${ }^{1}$ Dept. of Mathematics \\ King's College London \\ The Strand, London \\ WC2R 2LS, UK. \\ ${ }^{2}$ Theoretische Physik \\ Ludwig-Maximilians Universität \\ Theresienstrasse 27 \\ 80333 Munich \\ Germany.
}

ABSTRACT: In this talk we will discuss the low energy dynamics of non-BPS branes constructed as stable brane/anti-brane pairs at an orbifold. In particular we will determine the effective field theory and compare its predictions with those of the full open string theory. While the position and vector degrees of freedom of the branes have the familar form found in supersymmetric gauge theories, the massless modes originating in the tachyonic sector display novel non-commuting flat directions. We will show that these flat directions persist to all orders in $\alpha^{\prime}$. Finally we will briefly discuss the open string loop corrections.

\section{KEYwORDS: "non-BPS-branes, solitons, effective actions'}

\section{Introduction}

In the past two years Sen has pioneered the study of stable non-BPS branes in string theory. Here we consider stable non-BPS states constructed as brane/anti-brane pairs on an orbifold that removes any tachyonic instabilities. The existence of such states first appeared as a consequence of various string dualities. A natural question that arises with stable non-BPS branes is to obtain their low energy effective Lagrangian. Indeed there are several motivations for this study. The first is that in the case of BPS branes, most of their dynamics can be readily understood from the more familiar dynamics of the quantum gauge field theory living on their worldvolume. Indeed only the lowest order term in an $\alpha^{\prime}$ expansion of the effective Lagrangian is usually needed and this is none other than that of a maximally supersymmetric Yang-Mills theory. Therefore one might hope that by deriving the low energy effective theory for non-BPS branes one would find a simpler and more familiar description of their dynamics. On the other hand the lack of supersymmetry leads one to question how appropriate the low energy field theory approximation is.

Branes, most recently through the AdS/CFT correspondence, have allowed for radical and new approaches to the study of the strong coupling limit of supersymmetric field theories. One might think that non-BPS branes will open an analogous study for non-supersymmetric gauge theories. However there is a large obstacle to this approach. In the AdS/CFT correspondence a crucial role is played by the low energy supergravity solution for a large number of branes. As is well known, for BPS-branes there is a no-force condition and hence there is no limit to the number and location of the branes. However it was shown in $[1]$ in the the is a one loop repulsive force between two parallel stable non-BPS branes, except at the point in their moduli space where they become unstable. Hence an important question that remains is whether or not large numbers of non-BPS branes can somehow be prevented from flying apart so that a supergravity solution can 


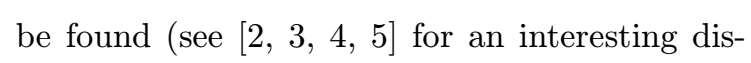
cussion of this point).

It is also very natural to suppose that nonBPS branes have an important role to play in phenomenological string models, since they have no worldvolume supersymmetry (although supersymmetry is preserved in the bulk). To our knowledge however there has not yet appeared any attempts in the literature along these lines.

In this talk we will review our study of the effective field theory for stable non-BPS branes $\overline{6_{1}}, \overline{7}, \bar{i}$ and also briefly report on some work in progress the literature as a whole or to provide explicit technical details, which are available in the original papers. Some more details may also be found in the talk of Janssen and Meessen in these proceedings [9]. Our plan is as follows. First we will provide a brief review of non-BPS. Secondly we will obtain the classical low energy effective Lagrangian for a stack of parallel non-BPS branes, to all orders in $\alpha^{\prime}$. We will do this for the specific case of a non-BPS brane at the critical orbifold radius, beyond which it becomes unstable. For a general radius of the orbifold the full effective Lagrangian is unknown and obtaining it appears to be a very challenging problem, although the lowest order term in $\alpha^{\prime}$ is known $\left[\hat{i}_{1}^{-i}\right]$. Thirdly we will provide a description of the classical moduli space of vacua and some of the topologically stable soliton states. Finally we will discuss the one loop quantum corrections to the effective Lagrangian. This will be done in in two ways; from the lowest order (renormalisable) term in the effective quantum field theory and also from the full open string theory. This allows us to compare the two predictions and test to what extent the quantum field theory approximation captures the full dynamics of the string theory.

\section{Review of Stable Non-BPS Branes Wrapped on $T^{4} / \mathbf{Z}_{2}$.}

We start by briefly recalling the key features nonBPS D $p$-branes as presented in the reviews articles $10_{0}^{\prime} 1 \overline{1} 1$. A non-BPS D $p$-brane (which we denote as a $\tilde{D} p$-brane) of type IIA (type IIB) string theory can be defined by starting with a $\mathrm{D} p$-brane/anti-D $p$-brane pair of type IIB (type
IIA) string theory. One then mods-out the theory by $(-1)^{F_{L}}$, which changes the sign of the left-moving spacetime Fermions. In the bulk this takes type IIB to type IIA string theory (or viceversa). In terms of the open strings ending on the $\mathrm{D} p$-branes this removes half of the Chan-Paton indices. In this way we see that a $\tilde{D} p$-brane has two types of open strings ending on it, labelled by the Chan-Paton indices $I$ and $\sigma_{1}$. Contrary to BPS D $p$-branes one has $p$ even in type IIB string theory and $p$ odd in type IIA string theory. The $I$-sector strings are precisely the same as for BPS D-branes and therefore their low energy modes form a maximally supersymmetric vector multiplet in $(p+1)$ dimensions. The $\sigma_{1}$-sector strings come with the opposite projection under $(-1)^{F}$. After the GSO projection, the level one sector modes of the NS-sector are projected out while the tachyonic ground state survives. Therefore the lightest modes in the $\sigma_{1}$-sector consist of real tachyonic scalar with mass-squared $-1 / 2 \alpha^{\prime}$ and a sixteen-component massless Fermion.

Clearly this system is unstable due to the tachyon. However by wrapping the brane around an orbifold, for example we will take $T^{4} / \mathbf{Z}_{2}$ with coordinates $i=6, \ldots, 9$ and radii $R_{i}$, the tachyonic modes which are even under the orbifold action $g: x^{i} \leftrightarrow-x^{i}$ are projected out. Thus only the tachonic modes with odd momentum around the orbifold survive. Since a momentum mode has mass-squared

$$
m^{2}=-\frac{1}{2 \alpha^{\prime}}+\sum_{i=6}^{9}\left(\frac{n_{i}}{R_{i}}\right)^{2},
$$

we see that below a critical radius $R_{c}=\sqrt{2 \alpha^{\prime}}$ the surviving states are non-tachyonic. In particular the four lightest tachyon scalars surviving the orbifold are given by

$$
T^{i}=\frac{\chi^{i}(x)}{2 i}\left(e^{i \vec{\omega}^{i} \cdot \vec{X}}-e^{-i \vec{\omega}^{i} \cdot \vec{X}}\right),
$$

where $\vec{\omega}^{i}=\left(R_{i}\right)^{-1} \vec{e}^{i}$. In this way we obtain a stable $\tilde{D} p$-brane.

On the other hand, the effect of the orbifold on the Bosons of the $I$-sector strings are the same as for a $\mathrm{D} p$-brane at an orbifold. Therefore, in the case of a $\tilde{D} 7$-brane wrapped on $T^{4} / \mathbf{Z}_{2}$, which we will be most interested in here ${ }^{1}$, the $N=4$

\footnotetext{
${ }^{1}$ Note that this is T-dual to the case considered in [6i $\left[{ }^{6}\right]$.
} 
vector multiplet is reduced to an $N=2$ multiplet. This contains two scalars $X^{I}, I=4,5$, that represent the fluctuations of the brane in the $x^{4}$ and $x^{5}$ directions, and a vector $A_{\mu}, \mu=0,1,2,3$. For the Fermions the orbifold selects out one chirality under $\Gamma^{6789}$ for the $I$-sector and the opposite for the $\sigma_{1}$-sector. This is the same Fermionic content as four-dimensional $N=4$ super-YangMills.

For most of this talk we will restrict our attention to oribifolds at the critical radius $R_{i}=$ $\sqrt{2 \alpha^{\prime}}$ where the lightest tachyon modes are massless. We will only use the Einstein summation convention for the full ten-dimensional indices $m, n=0,1,2 \ldots, 9$ and the four-dimensional indices $\mu, \nu=0,1,2,3$. All other sums (e.g. those over the $i, j$ and $I, J$ indices) will be explicitly written.

\section{The Effective Lagrangian for Par- allel non-BPS Branes}

In general it is a very hard problem to analyse the effect of relevant vertex operators on the string worldsheet. However, at the the critical radius the tachyon vertex operators are marginal. Indeed at this radius it is possible to Fermionise, and then re-Bosonise, these vertex operators into the form of Wilson lines [11 $\left.\overline{1}_{-}^{1}, 1_{2} \overline{3}\right]$ as follows. One starts by expressing the open string fields $X^{i}$ and $\psi^{i}$ in terms of the boundary values of left and right-handed closed string fields: $X^{i}=2 X_{L}^{i}=$ $2 X_{R}^{i}$ and $\psi^{i}=\psi_{L}^{i}=\psi_{R}^{i}$. Next one Fermionises these closed string fields as

$$
e^{i \sqrt{2 / \alpha^{\prime}} X_{L / R}^{i}}=\frac{1}{2}\left(\xi_{L / R}^{i}+i \eta_{L / R}^{i}\right) \otimes \Gamma^{i} .
$$

Here the $\Gamma^{i}$ matrices form a representation of the Spin(4) Clifford algebra. Furthermore the other worldsheet Fermions are now tensored with a $\Gamma^{5}$, e.g. $\psi_{L / R}^{i} \rightarrow \psi_{L / R}^{i} \otimes \Gamma^{5}$. These $\Gamma$-matrices arise as co-cycles and ensure that the worldsheet fields obey the correct statistics, i.e. that $\xi_{L / R}^{i}$ and $\eta_{L / R}^{i}$ commute with worldsheet Bosons but anticommute with worldsheet Fermions. Finally one can re-Bosonise by taking

$$
\frac{1}{2}\left(\xi_{L / R}^{i}+i \psi_{L / R}^{i}\right)=e^{i \sqrt{2 / \alpha^{\prime}} \tilde{X}_{L / R}^{i} \otimes \tilde{\Gamma}^{i},}
$$

where the $\tilde{\Gamma}$-matrices are another representation of the Spin(4) Clifford algebra. In this way we find an equivalent description of the worldsheet fields in terms of $X^{m}, \psi^{m}, \tilde{X}^{i}, \eta^{i}, m=0,1,2, \ldots, 5$, $i=6,7,8,9$. After this change of variables one can see that the tachyon vertex operators corresponding to the states $\overline{2} .2$ are mapped into Wilson lines but with the enlarged Chan-Paton factor $\chi^{i} \otimes \sigma_{1} \otimes i \Gamma^{5} \Gamma^{i}$. Here the $\sigma_{1}$ represents the fact that the tachyon comes from the $\sigma_{1}$-sector and the $i \Gamma^{5} \Gamma^{i}$ factor comes from the co-cycles.

The analysis for all the degrees of freedom is most easily presented after T-dualising in the $x^{4}$ and $x^{5}$-directions. In this case the positions of the branes are also given by Wilson lines. Thus, with the aid of these new variables, we now consider $N \tilde{D}$ 9-branes wrapped around $T^{4} / \mathbf{Z}_{2} \times T^{2}$, which are described by the Wilson line $\mathcal{A}_{m}, m=$ $0,1,2, \ldots, 9[\overline{7}]$

$$
\mathcal{A}_{m}=\left\{\begin{array}{l}
A_{\mu} \otimes I \otimes I ; \quad m=0, \cdots, 3 \\
\phi^{I} \otimes I \otimes I ; \quad m=4,5 \\
\chi^{i} \otimes \sigma^{1} \otimes i \Gamma^{5} \Gamma^{i} ; \quad m=6, \cdots, 9
\end{array}\right.
$$

where the fields $A_{\mu}, \phi^{I}, \chi^{i}$ all take values in the Lie algebra of $U(N)$.

We can now compute the effective Lagrangian for a $\tilde{D}$ 9-brane within the existing formalism for Wilson lines (for example see [i] ences therein). In general the effective Lagrangian will receive corrections to all orders in $\alpha^{\prime}$ and $\mathcal{F}_{m n}=\partial_{m} \mathcal{A}_{n}-\partial_{n} \mathcal{A}_{m}-i g\left[\mathcal{A}_{m}, \mathcal{A}_{n}\right]$ and we are unable to find an analytic expression. A simplification occurs if we neglect terms of the form $D_{(m} \mathcal{F}_{n) p}$, in which case we find [1 $\left.1 \overline{4}^{-}\right]$

$$
\mathcal{L}_{B}=c_{0} \mathrm{~S} \operatorname{Tr} \sqrt{\operatorname{det}\left(\delta_{m n}+2 \pi \alpha^{\prime} \mathcal{F}_{m n}\right)} .
$$

where $c_{0}$ is a constant and $\mathrm{STr}$ is the symmeterised trace. Note that the determinant here is taken over $m, n$ indices and the trace is over the Chan-Paton and co-cycle factors in $3 . \overline{3}$.

Next we may T-dualise and take the limit where $x^{4}$ and $x^{5}$ dimensions decompactify to obtain the effective Lagrangian for various $\tilde{D} p$-branes, viewed as $\tilde{D}(p+4)$-branes wrapped over the orbifold. Here we will concentrate on a $\tilde{D} 3$-branes. The lowest non-trivial term of $3 . \overline{4}$ gives a renormalisable effective Lagrangian

$$
\mathcal{L}_{B}=c_{0}^{\prime} \operatorname{Tr}\left(\frac{1}{4} F_{\mu \nu} F^{\mu \nu}+\frac{1}{2} \sum_{I} D_{\mu} \phi^{I} D^{\mu} \phi^{I}\right.
$$




$$
\begin{gathered}
\left.+\frac{1}{2} \sum_{i} D_{\mu} \chi^{i} D^{\mu} \chi^{i}-V\right) \\
V=\frac{g^{2}}{4} \sum_{I, J}\left(\left[\phi^{I}, \phi^{J}\right]\right)^{2}+\frac{g^{2}}{2} \sum_{I, j}\left(\left[\phi^{I}, \chi^{j}\right]\right)^{2} \\
-\frac{g^{2}}{4} \sum_{i \neq j}\left(\left\{\chi^{i}, \chi^{j}\right\}\right)^{2}
\end{gathered}
$$

where $c_{0}^{\prime}$ is a constant and $D_{\mu}=\partial_{\mu}-i g\left[A_{\mu},\right]$. Here we have dropped a constant term in $V$ and the trace is now only over the group indices $a, b$.

The lowest order terms in the low energy effective Lagrangian can also be obtained by taking the $\alpha^{\prime} \rightarrow 0$ limit of the disk diagrams for the open string S-matrix [6]. The Bosonic part of the resulting effective action agrees precisely with 3.5 However in addition the couplings of the (massless) Fermionic fields on a $\tilde{D} p$-brane can be determined $[\overline{\underline{6}} \mid$. If we denote the Fermions arising the $I$-sector and $\sigma_{1}$-sectors by $\lambda$ and $\psi$ respectively then, to lowest order, the total effective Lagrangian has the form $\mathcal{L}=\mathcal{L}_{B}+\mathcal{L}_{F}$ where

$$
\begin{aligned}
\mathcal{L}_{F}= & c_{0}^{\prime} \operatorname{Tr}\left(i \bar{\lambda} \gamma^{\mu} D_{\mu} \lambda+i \bar{\psi} \gamma^{\mu} D_{\mu} \psi\right. \\
& \left.+g \bar{\lambda} \gamma^{I}\left[\phi^{I}, \lambda\right]+g \bar{\psi} \gamma^{I}\left[\phi^{I}, \psi\right]\right)
\end{aligned}
$$

Note that there are no Yukawa interactions involving the four scalars $\chi^{i}$. Thus, even though at the critical radius where we have exactly the same field content as a maximal Yang-Mills multiplet, the effective Lagrangian is not supersymmetric. Therefore one can expect that there will be non-trivial loop corrections in both the string theory and field theory approximation.

The string S-matrix also allows one to work away from the critical radius, where the modes $\chi^{i}$ have a mass $m_{i}^{2}=1 / R_{i}^{2}-1 / 2 \alpha^{\prime}$. In this case the only change to the Lagrangian is the appearance of a mass term $\frac{1}{2} m_{i}^{2} \chi_{i}^{2}$ in $V$ [i,i]. Note that if the $\tilde{D} p$-brane is unstable, so that at least one $m_{i}^{2}$ is negative, then the potential $V$ is unbounded from below since we may make the corresponding $\chi^{i}$ as large as we wish with the other fields vanishing and $V$ will then be arbitrarily negative. Therefore the lowest order (renormalisable) term in the effective Lagrangian is not a valid description for unstable $\tilde{D} p$-brane.

\section{The Classical Vacua and BPS States of Non-BPS Branes}

Perhaps the most notable feature of the effective Lagrangian 3.51 is that the classical vacuum moduli space, given by the space of constant scalars, has non-Abelian flat directions. Indeed we can see that the condition for constant $\chi^{i}$ and $\phi^{I}$ scalar vertex operators to be marginal is $\left[\mathcal{A}_{m}, \mathcal{A}_{n}\right]=$ 0 or, more explicitly,

$$
\left[\phi^{I}, \phi^{J}\right]=\left[\phi^{I}, \chi^{i}\right]=\left\{\chi^{i}, \chi^{j}\right\}=0,
$$

for $i \neq j$ and to all orders in $\alpha^{\prime}$. Of course since there is no supersymmetry we do not expect the situation to persist once string loops are taken into account.

We also note that, in a vacuum parameterised by anti-commuting vev's for $\chi^{i}$, the gauge group can be broken down to a single $U(1)$ representing the centre of mass, rather than $U(1)^{N}$ as is the case for vacua in which the scalars $\phi^{I}$ have a nonvanishing vev. In addition the components of $\phi^{I}$ which are not in this $U(1)$ will become massive. Thus, since the $\phi^{I}$ describe the separation of the branes, we see that in these vacua the non-BPS branes are classically bound together. Clearly if $m_{i} \neq 0$ then the $\chi^{i}$ vev's must vanish.

Let us now mention some of the soliton solutions on the $\tilde{D} 3$-brane worldvolume. Although one can find several types of BPS states of the Lagrangian $3 . \overline{5} \cdot \overline{7} \cdot \overline{1}$, here we will concentrate on the monopoles. If we turn on just one scalar, either $\chi^{i}$ or $\phi^{I}$, then there is no potential to all orders in $\alpha^{\prime}$ and the Bosonic Lagrangian is identical to that obtained from a maximally supersymmetric D3-brane. It then follows that the BPS monpoles given by the first order equations $B_{a}=D_{a} \phi^{I}$ or $B_{a}=D_{a} \chi^{i}(a=1,2,3)$, where $B_{a}=\frac{1}{2} \epsilon_{a b c} F^{b c}$ is the magnetic field, are absolute minimum energy configurations for a fixed topological index given by the magnetic charge. Therefore, even without supersymmetry, we expect these states to be stable.

It is well known that on a D3-brane a monopole configuration involving the scalar $\phi^{I}$ corresponds to a D1-brane suspended between two D3-branes along the $x^{I}$ direction. A similar interpretation occurs here expect that, since we are in type IIA string theory, it must be a $\tilde{D} 1$-brane suspended 
between two $\tilde{D} 3$-branes. On the other hand a tachyon vev $\chi^{i}=1$ corresponds to deforming a single wrapped $\tilde{D} 7$-brane into a D6-brane and an anti-D6-brane wrapped over the $x^{6}, x^{7}, x^{8}$ directions but sitting at opposite ends of the orifold [1010']. Therefore a monopole soliton involving $\chi^{i}$ can be pictured as two wrapped $\tilde{D} 7$-branes at the monopole core, but at infinity the two $\tilde{D} 7$-branes split up into D6-brane/anti-D6-brane and antiD6-brane/D6-brane pairs at opposite ends of the orbifold. These states again correspond to $\tilde{D} 1$ branes but this time stretched along an orbifold direction $x^{i}$.

Now consider what happens if we move away from the critical radius. In this case the a mass term $\frac{1}{2} m_{i}^{2} \chi_{i}^{2}$ appears in the Lagrangian and so the $\chi^{i}$ monopoles no longer exist. This can be understood because a $\tilde{D} 3$-brane is stable and hence the $m_{i}^{2}$ are positive only if the orbifold radii are larger than the critical radius (note that we are in the T-dual picture compared to section two). However a $\tilde{D} 1$-brane stretched along an orbifold direction is stable only if the radius is less than critical.

\section{Quantum Corrections}

Finally we would like to discuss the quantum corrections to the classical effective Lagrangian described above. We will do this in two ways. First we will calculate the one loop quantum corrections in the full string theory consider the renormalisable quantum field theory given by the lowest order terms in the effective Lagrangian to test how well it reproduces the string theory results. For simplicity we consider the case of two $\tilde{D} 3$-branes.

\subsection{Open String Theory}

It was shown in [i] that away from the critical radius, where they are stable, there is a repulsive force between two parallel $\tilde{D} p$-branes at any separation. At the critical radius the force vanishes because there is a spurious Bose-Fermi degeneracy, at all levels in the open string theory, and so the one loop effective potential (which is the negative of the partition function) vanishes.
We have seen above that at the critical radius there a new branches of the classical vacuum moduli space associated with

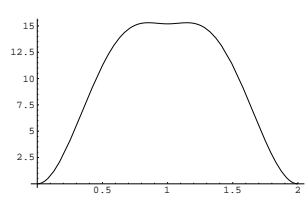
non-Abelian flat directions. Figure 1: $V_{\text {eff }}$ as a Here we will report on some function of $v_{0}$ work in progress to deter-

mine the partition function in these branches [i, There are two cases that are of interest. Either $\left\langle\chi^{9}>=v_{0} I\right.$ or $\left\langle\chi^{9}>=v_{3} \sigma_{3}\right.$, where $\sigma_{3} \in s u(2)$. More complicated vacua, with more than one tachyon non-vanishing can also be considered but the calculation becomes increasingly complicated and we do not expect the general form to change. The plots for the effective potential for these two cases are given in figures 1 and 2 respectively.

Note that these plots are periodic in $v_{0}$ with period 2 and periodic in $v_{3}$ with period 1 . In both of these cases there is a divergence in the integral over the open string modular parameter. These are

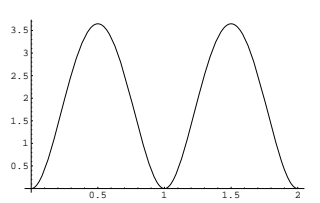

Figure 2: $V_{\text {eff }}$ as a function of $v_{3}$ related to the existence of tadpoles diagrams for the closed string fields via the Fischler-Susskind mechanism ["].].].

Turning on a vev $<\chi^{9}>=1$ on wrapped $\tilde{D} 7$-branes corresponds to deforming all of them into D6-brane/anti-D6-brane pairs at opposite ends of the the orbifold, i.e. the D6-branes are at one end point and the anti-D6-branes at the other. We see from figure 1 that this configuration is stable only up to very small fluctuations. The true minima is at $\left\langle\chi^{9}\right\rangle=0$. One the other hand turning on a non-Abelian vev $<\chi^{9}>=\sigma_{3}$ is a true minima. It correponds to spliting up one $\tilde{D} 7$-brane into a D6-brane/antiD6-brane pair and the other into an anti-D6brane/D6-brane pair, i.e. there are equal numbers of D6-branes and anti-D6-branes at each end point.

\subsection{Quantum Field Theory}

Lastly let us consider the one-loop effective potential for the scalars by treating 3.5 as a quantum field theory. If $m_{i}=0$ then the Bose-Fermi 
degeneracy ensures that the effective potential vanishes at one-loop. However since the interactions are not supersymmetric we do not expect a cancellation at higher loops. For $m_{i} \neq 0$ it is not hard to calculate the effective potential for a scalar vev $\left\langle\phi_{I}\right\rangle=v_{I} \sigma_{3}$ [i $\overline{1}$. The result has the classic Coleman-Weinberg form given in figure 3 . There we included several plots which correspond to adding various mass renormalisations since, in the absence of supersymmetry, there appears to be no principle to exclude them.

Since the $v_{I}$ parameterise the relative separation of the branes, $V_{e f f}\left(v_{I}\right)^{\circ}$ is the potential between two branes as a function of their distance. So we see that for small separations there is a repulsive

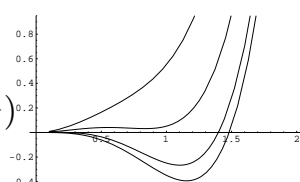
Figure 3: $\quad$ The Coleman-Weinberg form for $V_{\text {eff }}$ force between the branes (at least for small or zero values of the renormalised mass), but it has a stable minimum and is attractive for large separations.

We should, however, question the validity of the field theory approximation. Generally speaking the one-loop approximation in quantum field theory is only valid for large $v_{I}$ but in our case this is where the masses of the low energy fields

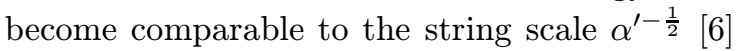
and so the low energy approximation is no longer valid. Therefore the region where the force is attractive should not be trusted. Indeed we know from the full string theory that the one loop force is repulsive everywhere $v_{I}$ region is strictly speaking not reliable in quantum field theory one can use renormalisation group methods to gain a reliable form. Since we do find a repulsive force there, in agreement with [i], we expect that the basic form for the effective potential is unaltered by renormalisation group techniques.

Now let us return to the case of $m_{i}=0$ where there are non-Abelian flat directions and we consider the two cases: $\left\langle\chi^{9}\right\rangle=v_{0} I$ and $<\chi^{9}>=v_{3} \sigma_{3}$. In either case the one loop effective potential $V_{\text {eff }}\left(v_{0}\right)$ or $V_{\text {eff }}\left(v_{3}\right)$ again takes on the classic Coleman-Weinberg form of figure 3 [i, However again we can not trust the large $v_{0}, v_{3}$ region. We see from figures 1 and 2 that string theory predicts a non-zero renormalised mass.

In summary it would appear that the effective potential for $\tilde{D} p$-branes provides an good, and hopefully helpful, description at the classical level. However the one-loop corrections that appear in the quantum field theory, are only valid for small scalar vev's and depend on a priori undetermined mass counter terms which are, in turn, fixed in string theory.

\section{Acknowledgements}

The work of N.D.L. has been supported by the EU grant ERBFMRX-CT96-0012, the PPARC grant PA/G/S/1998/00613 and also a PPARC Advanced Fellowship.

\section{References}

[1] M.R. Gaberdiel and A. Sen, JHEP 11 (1999) 008, hep-th/9908060.

[2] E. Eyras and S. Panda, hep-th/0003221.

[3] Y. Lozano, hep-th/0003226.

[4] P. Brax, G. Mandal and Y. Oz, hep-th/0005242.

[5] M. Bertolini, P. Di Vecchia, M. Frau, A. Lerda, R. Marotta and R. Russo, hep-th/0007097.

[6] N.D. Lambert and I. Sachs, JHEP 05 (2000) 200, hep-th/0002061.

[7] N.D. Lambert and I. Sachs, hep-th/0006122.

[8] N.D. Lambert, I. Sachs and G.M.T. Watts, in preparation.

[9] B. Janssen and P. Meessen, hep-th/0009025.

[10] A. Sen, hep-th/9904207.

[11] M.R. Gaberdiel, hep-th/0005029.

[12] A. Sen, JHEP 09 (1998) 023, hep-th/9808141.

[13] J. Majumder and A. Sen, hep-th/0003124.

[14] A. Tseytlin, hep-th/9908105. 